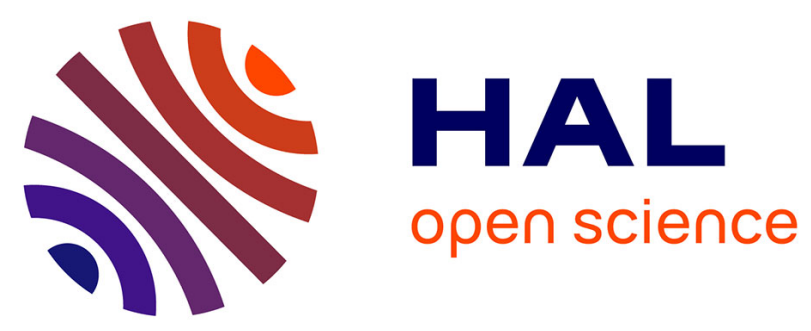

\title{
Diffuser une alerte aux crues rapides via une application Smartphone en France: de la théorie à la mise en pratique
}

Sekedoua Kouadio, Johnny Douvinet

\section{- To cite this version:}

Sekedoua Kouadio, Johnny Douvinet. Diffuser une alerte aux crues rapides via une application Smartphone en France: de la théorie à la mise en pratique. Revue des Sciences et Technologies de l'Information - Série ISI: Ingénierie des Systèmes d'Information, 2016, DIFFUSION D'ALARMES, DIFFUSION D'ALERTES DE NOUVEAUX ENJEUX POUR LES SI, 21 (4), pp.4966. 10.3166/ISI.21.4.49-66 . halshs-01485258

\section{HAL Id: halshs-01485258 \\ https://shs.hal.science/halshs-01485258}

Submitted on 8 Mar 2017

HAL is a multi-disciplinary open access archive for the deposit and dissemination of scientific research documents, whether they are published or not. The documents may come from teaching and research institutions in France or abroad, or from public or private research centers.
L'archive ouverte pluridisciplinaire HAL, est destinée au dépôt et à la diffusion de documents scientifiques de niveau recherche, publiés ou non, émanant des établissements d'enseignement et de recherche français ou étrangers, des laboratoires publics ou privés.

\section{(ㄷ)(1) $\$$}

Distributed under a Creative Commons Attribution - NonCommerciall 4.0 International 


\title{
Diffuser une alerte aux crues rapides via une application smartphone en France
}

\section{De la théorie à la mise en pratique}

\author{
Sekedoua Kouadio $^{1,2}$, Johnny Douvinet ${ }^{1,3}$ \\ 1. UMR ESPACE 7300 CNRS, Université d'Avignon et des Pays de Vaucluse \\ 74 rue Louis Pasteur, Case 19, 84029 Avignon Cedex 1, France \\ 2. Université de Reims, 9 bld de la Paix, 51100 Reims \\ 3. FR Agorantic 3621 CNRS, Université d'Avignon et des Pays de Vaucluse \\ 74 rue Louis Pasteur, Case 19, 84029 Avignon Cedex 1, France \\ sekedoua-jules.kouadio@univ-reims.fr,johnny.douvinet@univ-avignon.fr
}

RÉSUMÉ. Cet article s'intéresse aux conditions nécessaires à la mise en œuvre d'un système spécifiquement basé sur une application Smartphones en vue de contribuer à l'amélioration du dispositif actuel d'alerte face aux crues rapides dans les petits bassins versants $\left(<50 \mathrm{~km}^{2}\right)$. Il s'agit surtout de savoir si un tel outil pourrait 1) réduire le temps nécessaire à l'alerte et à la protection des populations, 2) valoriser la collecte d'informations émanant des citoyens à l'échelle locale (dans une approche «bottom-up »), 3) faciliter la mise en place d'une vigilance entre les citoyens eux-mêmes en créant une sorte de "réseau sentinelles» indépendamment des services de l'Etat et 4) améliorer les connaissances sur les dommages observés en temps réel, alors que la vigilance actuelle se focalise principalement sur l'aléa hydrologique. La solution envisagée doit être pensée pour réduire les écarts entre une approche étatique régalienne, qui positionne et impose l'État comme principal lanceur officiel d'alerte, et une action citoyenne qui érige l'individu au rang de "citoyen capteur », capable de remonter ou de diffuser une alerte à travers l'usage de son Smartphone. Répondre à ce défi ne s'avère toutefois pas si simple au regard des contraintes juridiques et techniques préexistantes en France.

ABSTRACT. This paper aims at developing a new Smartphone application to further assess the actual warning system to face to flash floods occurring in France in small basins (of less than $50 \mathrm{~km}^{2}$ ). We mainly discuss in this paper of the condition of the establishment of a new system using the reactiveness of Smartphones. We hope 1) to reduce the time required for warnings and protect population; 2) to enhance data collection provided by citizens since local scales; 3) to built a collaborative vigilance and a standing guard network based on these "citizenssensors" not depending on the official government systems; 4) to improve knowledge on flash floods damage observed in real-time. The solution is thought in the way to limit drawbacks between the classical approach (top-down) that imposed the government as a main warning source, and the citizens (bottom-up) we consider as "sensors" during crisis (both in going up 
or disseminating warning information). Addressing this is of paramount importance if we aim at minimizing material \& human damages regarding the high acuity of flash floods.

MOTS-CLÉS : smartphone, applications, géolocalisation, crues rapides, citoyen capteur.

KEYWORDS: smartphone, application, geo-localisation, flash floods, sensor citizen.

DOI:10.3166/ISI.21.4.49-66 @ 2016 Lavoisier

\section{Introduction et contexte de recherche}

En France, les crues rapides sont à l'origine d'une part significative des dégâts matériels (500 millions $€$ dans l'Aude en 1999, 1,2 milliard $€$ dans le Gard en 2002, plus d'un milliard $€$ dans le Var en 2010) et sont responsables d'une grande partie de la mortalité (26 morts le 15 juin 2010, 6 morts en septembre 2014, 20 morts le 3 octobre 2015) enregistrée au cours de ces dernières années (Jonkman et Kelman, 2005 ; Ruin et al., 2007 ; Vinet, 2010 ; Morss et al., 2016). Générées par des pluies intenses (avec des cumuls pouvant dépasser $600 \mathrm{~mm}$ en $24 \mathrm{~h}$ dans le Sud-Est de la France), ces crues se manifestent au sein de petits bassins versants $\left(<50 \mathrm{~km}^{2}\right)$ dont les temps de réactions sont courts (de $1 \mathrm{~h}$ à $6 \mathrm{~h}$ ) et les débits spécifiques élevés (allant jusqu'à $20 \mathrm{~m}^{3} / \mathrm{s} / \mathrm{km}^{2}$ ). Face à ces aléas, le ministère de l'Environnement (MEDDE) a lancé en 2011 le Plan submersions rapides (abrévié PSR). Ce plan prévoit entre autres le développement d'un service d'avertissement spécifique, et la mise en place par le Schapi (Service central d'hydrométéorologie d'appui à la prévision des inondations) est actuellement à l'étude. Un premier service (VigicruesFlash), basé sur le déploiement du modèle AIGA-Hydro pour l'ensemble de la France, devrait être en œuvre en 2016 pour couvrir pas moins de 3000 bassins de taille intermédiaire (> $\left.10 \mathrm{~km}^{2}\right)$ correspondant aux secteurs les plus vulnérables.

Ce système permettra une qualification de l'ampleur des réponses hydrologiques à attendre au regard de l'intensité de la pluie observée (en temps réel) et servira aux gestionnaires de crise. Un tel système présente toutefois des limites et des évolutions sont nécessaires au regard :

- du nombre élevé de cours d'eau pouvant générer des crues rapides (300.000 $\mathrm{km}$ de talwegs drainent des surfaces de plus de $5 \mathrm{~km}^{2}$ d'après la Base nationale de bassins versants du Schapi),

- de l'absence d'instrumentations sur ces petites entités par manque de moyens et d'intérêt (Douvinet et al., 2011),

- du manque d'estimation sur les impacts directs et indirects sur les personnes et les biens (Saint-Martin, 2014 ; Douvinet et al., 2016),

- des incertitudes engendrées par les évolutions climatiques, qui modifient les périodes de retour des aléas et qui font craindre une augmentation du nombre de victimes, ce chiffre ayant tendance à augmenter chaque année (Jonkman et Kelman, 2005 ; Ruin et al., 2007 ; Morss et al., 2016), 
- de l'obsolescence des systèmes d'alarme et du Réseau national d'alerte (qui date de 1952 et qui doivent être modernisés pour 2017),

- du manque d'exercices réguliers en amont des catastrophes,

- de la surabondance des informations diffusées par les médias pendant et après une crise.

Aujourd'hui, très peu de citoyens (entre 5 et $30 \%$ ) savent comment adapter leur comportement en situation de risque (Creton-Cazenave, 2008). Face à un tel constat, cette recherche propose d'utiliser les Smartphones et d'exploiter leurs capacités pour aider les populations à savoir quand, où et comment elles seront en danger. Cette approche se justifie d'autant plus au regard de l'expansion observée ces dernières années sur l'usage et les pratiques via de tels outils. En France, plus de 30 millions de personnes détiennent un smartphone et $80 \%$ des téléphones vendus aujourd'hui sont des smartphones (MMA, 2015). De tels développements (depuis la création du premier « téléphone intelligent » Simon par IBM en 1992) bouleversent la société en profondeur (Laurila et al., 2013 ; Slingsby et al., 2013 ; Woyke, 2014 ; Smith et al., 2015) et le nombre de connections mobiles (plus de 7 milliards en 2014 d'après la Banque mondiale) sont des chiffres qui sont tout aussi impressionnants et qui résument l'engouement suscité par ces outils, faisant désormais partie intégrante du quotidien (Gupta et al., 2013). Les utilisateurs de téléphones mobiles peuvent aujourd'hui être géo-localisés en temps réel et ainsi espérer avoir accès aux réseaux de secours incluant des messages d'alerte.

Il convient néanmoins de rester "prudent » et de ne pas surévaluer les attentes : il faut en effet rendre compréhensible les messages d’alerte (pour ne pas générer des paniques ou des comportements qui ajouteraient de la confusion dans l'intervention des services de secours) et inciter les populations à contribuer et à participer tout en ayant une flexibilité territoriale et une identification locale des zones à risque (Creton-Cazenave, 2010 ; Ruin et al., 2011). D'autres questions de recherche sont également sous-jacentes : 1) Comment gérer les données personnelles sans franchir la limite de la vie privée ? En France, seuls le gouvernement et les services de l'État (préfectures et mairies) détiennent le droit d'alerter (loi de 1982) et d'informer les populations concernant le risque inondation. 2) Quelle architecture matérielle et logicielle déployer pour ne pas dépendre des compagnies téléphoniques et/ou des réseaux de télécommunication ? 3) Puisque le système actuel fonctionne dans une approche «top-down » (« l'État alerte et la population réagit»), comment peut-on encourager les citoyens à s'engager dans une action citoyenne tout en évitant de produire le même schéma à un échelon local (autrement dit, ne pas donner un poids plus important aux citoyens qui seraient les lanceurs d'alerte donc considérés comme des donneurs d'ordre)?

Avant de développer une application (qui complètera la longue liste de celles qui existent déjà sur le marché, puisque plus de 350000 applications sur smartphones ont été créées dans de nombreux domaines en juin 2015), il semble nécessaire d'en évaluer les fondements et les limites et de lister des objectifs précis, d'autant plus 
que les applications spécifiques au risque inondation demeurent rares en France. Cet article présente les contraintes de faisabilité liées à la réalisation des applications Smartphone dans le domaine de l'alerte face aux inondations (section 2) puis les contraintes liées au cadre réglementaire (section 3), avant de présenter l'architecture de la nouvelle application proposée (section 4) et les expérimentations en cours.

\section{Les applications face au risque inondation : une voie encore peu explorée}

\subsection{Bref historique sur l'avènement des Smartphones}

Selon l'Institute of Electrical and Electronics Engineers (IEEE) un Smartphone est un téléphone mobile doté des capacités d'un ordinateur, lui permettant d'interagir avec d'autres systèmes informatiques. Il n'existe pas de définition officielle d'un « Smartphone », sauf la très populaire référence au « téléphone intelligent », ce qui devient vite réducteur car tous les téléphones sont intelligents dans la mesure où l'homme ne peut intervenir à tous les niveaux de leur fonctionnement (ce ne sera plus valable dans quelques années). En plus de sa fonctionnalité originelle, c'est-àdire pouvoir émettre ou recevoir des appels téléphoniques, cet appareil est en mesure d'effectuer des tâches initialement réservées aux assistants numériques personnels (PDA). Cet ordinateur a aussi la faculté de gérer et de traiter des informations issues des différents capteurs intégrés (accéléromètre, connexion internet, wifi, GPS, etc.) ou qu'il est possible d'amarrer à l'appareil en fonction des usages que l'on veut en faire (navigation, distraction, assistance, cartographie, géolocalisation, etc.). Doté d'un espace mémoire de plus en plus important, il est aussi possible de leur ajouter de nouvelles fonctionnalités, soit en les appareillant à des capteurs extérieurs, soit en y installant de nouvelles applications, soit en supprimant/désinstallant celles fournies au départ par le constructeur. Ces avantages permettent de convertir le smartphone en un appareil hautement personnalisable.

Les fonctionnalités des Smartphones ont considérablement évolué au cours des dernières années (figure 1). Après l'arrivée de Simon en 1992, qui fut soldée par un échec commercial, le marché du Smartphone va être dominé pendant une dizaine d'années par Nokia, avec sa série Communicator, sorte d'appareils pliants à deux écrans dotés de clavier physique complet. Avant 2006, les constructeurs gardaient le contrôle total sur le contenant et le contenu. Les utilisateurs en étaient dépendants et ils devaient attendre la sortie d'une nouvelle version pour avoir ces nouvelles fonctionnalités, ou attendre que ces fonctionnalités soient disponibles sur le site de la firme. Avec l'arrivée de Google et Apple en 2007, le Smartphone change de configuration (entraînant la chute de Nokia) en permettant à tout individu de passer du statut de consommateur à celui de producteur : toute personne motivée peut créer du contenu (application) et du contenant (informations) et les mettre, en l'espace de quelques minutes, à la disposition du grand public sur une boutique en ligne. Ce changement de pratique est facilité par l'introduction d'autres appareils, de plus en plus ubiquitaires et interconnectables, avec lesquels ils inondent le marché (tablettes, 
télévisions numériques, appareils photo équipés de GPS, etc.), mais également par l'avènement de réseaux sociaux numériques et la consumérisation des entreprises (FC, 2015). La démocratisation de la technicité, l’ouverture des bibliothèques de codes et la mise à disposition d'outils de développement favorisent la naissance de communautés d'utilisateurs : des contributeurs volontaires, passionnés, amateurs ou professionnels animant désormais la vie de cet univers et le font grandir chaque jour un peu plus. Le Smartphone symbolise dorénavant un bouleversement des habitudes et des relations interpersonnelles et familiales, rendant de plus en plus mince la frontière entre vie privée, vie publique et vie professionnelle (O’Reilly, 1996 ; Lemmens et al., 2009 ; Chak et Leung, 2004 ; Lapointe et al., 2013).

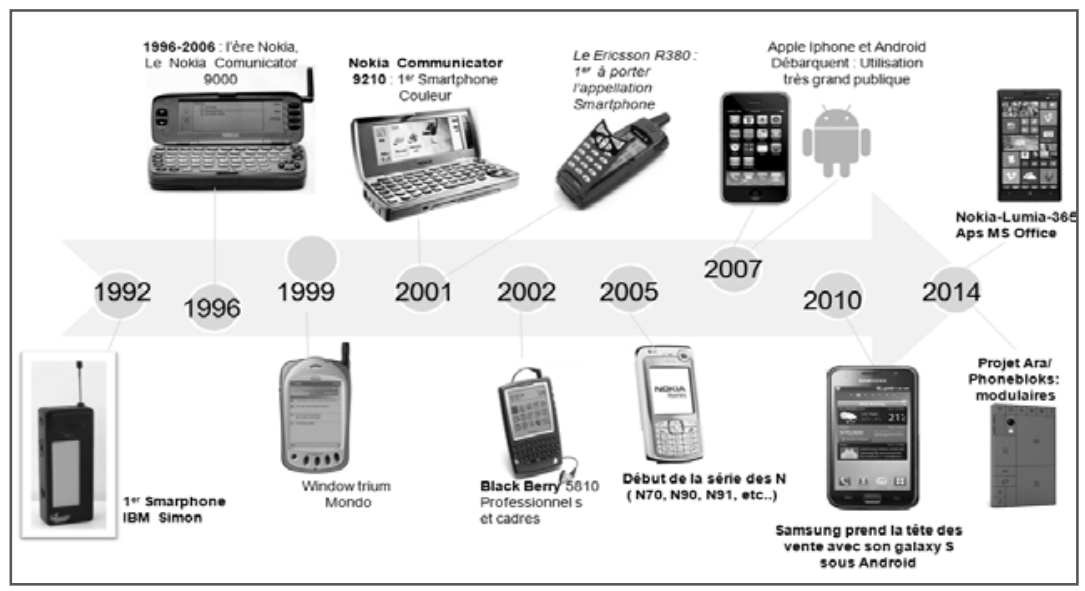

Figure 1. Évolution chronologique des modèles de smartphone

\subsection{Un nombre limité d'applications pour faire face au risque inondation}

Plus de 350000 applications existent aujourd'hui sur le marché (2015) mais un faible nombre est dédié spécifiquement au risque inondation. Un premier inventaire réalisé sur 17 applications (Kouadio et al., 2014) a permis de les dissocier en deux catégories. Des applications ont un caractère consultatif, permettant à l'utilisateur de visualiser des informations et d'avoir accès à des instructions liés à un danger (sans être associées à la survenue d'un événement en temps réel) ; c'est le cas par exemple pour FloodWatch (développé sous iOS6) ou FloodMap (Symbian OS). D’autres ont un caractère bien plus participatif, en offrant la possibilité de localiser le danger et en étant aussi consultatives ; l'utilisateur est alors un véritable « citoyen-capteur » (Goodchild, 2007) car il déclenche l'alerte et peut en être averti ; c'est par exemple le cas de Risques-Nice, développée par la ville de Nice, ou NOAH, promue par le gouvernement philippin (les deux étant disponibles sous Android et iOS). La décision d'alerter ou d'avertir revient ici à un centre de commandement rattaché à un dispositif administratif renforcé (mairie, conseil régional, État). 
Certaines applications ont fait l'objet de développement dans des structures privées. Signalert est par exemple une application dont la conception et le mode de fonctionnement s'inspirent de l'usage de marqueurs et d'échelles d'intensité qui sont graduelles, ce qui permet une description fine, détaillée et en temps réel des crues observées. Les informations sont gérées sur une plateforme web (où des conseils de comportement sont indiqués) et des alertes peuvent être envoyées par une autorité (aux détenteurs de l'application) dans un périmètre délimitable. Dans sa version payante, il est aussi possible de demander une assistance d'urgence, de contacter les autres observateurs pour un même événement ou de définir les secteurs à surveiller, c'est-à-dire les espaces sur lesquels on veut recevoir des alertes. Ce système est aussi doté d'un module d'exportation de données statistiques et cartographiques pour une réutilisation dans des systèmes annexes, grâce à l'intégration de systèmes de notifications automatiques nationaux et internationaux.

D’autres applications sont nées grâce à des partenariats public-privé. L’interface MyPublicAlerts (créée par la société Deveryware) et l'application Notico sont par exemple deux livrables d'un projet européen (ISAR+ FP7SEC2012-1). Elles visent trois objectifs : faciliter le dialogue entre les autorités et le grand public ; fédérer dans une approche systémique les compétences des autorités, les interactions Humain-application et l'ingénierie technique ; fournir une application multi-usage transcendant la question des risques. De son côté, la ville de Nîmes a, elle, associé une notification « push » dans l'application officielle de la ville pour informer les administrés d'une vigilance ou d'un niveau d'alerte. Ce système permet de recevoir une information sans en être à l'initiative. La ville a défini sa propre échelle de vigilance (de 0 à 4 ) et les couleurs sont différentes du site www.vigicrues.fr pour éviter toute confusion. L’information s’adapte à des niveaux prédéfinis par la ville et toutes les phases de la gestion de l'urgence (avant, pendant et après une crue rapide par exemple) s'accompagnent de messages intégrés dans un référentiel (niveau des eaux attendus, type de crise, niveau de gravité, comportement). Divers scénarios ont été imaginés pour laisser le moins de place possible à l’improvisation.

L’analyse comparative menée sur ces applications confirme que la majorité des applications doivent être préinstallées sur les téléphones et qu’elles dépendent du réseau internet avant d'être efficaces. Elles diffèrent aussi selon le mode de diffusion (horizontal, vertical ou les deux), du choix opéré dans l'utilisateur principal, dans les actions ciblées (alerte, avertissement, expertise), dans la couverture géographique, dans les risques étudiés... Elles sont donc peu nombreuses et peu transposables. En effet, ces applications ont été réalisées dans différents contextes et avec des objectifs tous aussi différents, ce qui contribue à limiter leur fonctionnement au-delà de leur zone de couverture d'expérimentation. Ces applications contribuent essentiellement à une prévention globale sans véritablement encourager un comportement particulier (comme ne pas utiliser sa voiture ou aller chercher les enfants, ce qui est une des consignes de sécurité en cas d'inondations, d'autant plus à respecter que la mobilité est la première cause de mortalité en cas de crues rapides (Antoine et al, 2001 ; Ruin et al., 2008). Réussir l'implémentation d'une nouvelle application demande aussi 
une bonne connaissance de l'environnement dans lequel elle sera déployée et de ses capacités à répondre aux besoins de ses utilisateurs. Trop souvent, le développement d'une application demande des ressources substantielles (aussi bien humaines que financières) et des autorisations spécifiques. Au-delà de ces premiers éléments, on se rend aussi vite compte que les développements sont limités, notamment en France, par des contraintes réglementaires et juridiques pesantes qui vont être plus détaillées et explicitées dans la section suivante.

\section{L’importance des contraintes réglementaires et juridiques en France}

\subsection{Les services étatiques contrôlent l'alerte}

En France, l'alerte est un signal donné pour informer une personne et/ou une population d'un danger imminent. Le décret $\mathrm{N}^{\circ} 2005-1269$ (du 12 octobre 2005) concernant le code national de l'alerte décrit les mesures à prendre pour alerter et informer la population en toute circonstance quelle que soit la menace ou l'agression au sens des articles L. 1111-1 et L. 1111-2 du Code de la défense, ou dans le cas d'un accident, ou d'une catastrophe au sens de la loi du 13 août de 2004. Déclencher cette alerte est donc une tâche à la fois délicate et vitale pour les maires (car relevant du leur pouvoir de police), les préfets ou les sous-préfets ou même le gouvernement français si l'alerte va au-delà des frontières départementales. Cette procédure demande des contrôles à plusieurs échelons avant la moindre validation. Sur la base des relevés fournis par les capteurs hydrométriques et pluviométriques, Météo France et le Schapi informent en premier lieu l'un des 19 SPC (Services de Prévision des Crues) en cas de probabilité élevée d’occurrence d'un évènement intense (associé à un niveau d'endommagement probable plus ou moins élevé).

Cette information est ensuite relayée aux services gouvernementaux (mairies et préfectures) et au public (via le site http://www.vigicrues.gouv.fr/index.php). Le site est actualisé tous les jours à 8 h et à 14 h UTC. Lorsque le niveau de vigilance passe du vert («pas de vigilance particulière requise ») à un niveau supérieur - jaune ( «risque de crue nécessitant une vigilance particulière »), orange (« risque de crues susceptibles d'avoir un impact significatif ») ou rouge (« risque de crue majeure ») le Centre interministériel de crise (CIC) est directement informé et relaie ensuite cette information aux acteurs locaux (SDIS, maire, police, association). Dans tous les cas ce système suit une approche top-down : l'administration informe le citoyen. Elle détient exclusivement le pouvoir d'information lors des évènements et le maire reste le premier responsable officiel de la sécurité des biens et des personnes dans la zone sinistrée (Douvinet et al., 2011). Une telle procédure apparaît alors très fortement technocratique et semble dépassée au regard des pratiques numériques actuelles. De nos jours, peu de citoyens associent le son des sirènes à un comportement spécifique à avoir en cas de catastrophe naturelle. A contrario, ils sont constamment sur leurs Smartphones ou connectés sur les réseaux sociaux. Les citoyens mettent souvent la question des risques loin de leurs préoccupations 
quotidiennes et cela s'explique, entre autres raisons, par le manque d'exercices pour faire face à l’imprévisible.

\subsection{Les services étatiques contrôlent l'utilisation des données personnelles}

Bien que populaire et bien accueilli, le Smartphone est encore considéré comme un appareil dont l'usage peut soulever des questions sécuritaires. Une enquête de la Commission nationale de l'informatique et des libertés (CNIL, 2013) a démontré que la majorité des applications accèdent en permanence (ou dès qu'elles en ont l'occasion) à la mémoire interne du téléphone et à la liste de contacts, qu'elles lisent et modifient des données, qu'elles accèdent aux données du GPS et transmettent tout cela via internet sans consentement. Des chercheurs ont par ailleurs montré que la société Apple enregistrait à leur insu la localisation des détenteurs d'un smartphone grâce à un système de triangulation (via les points d'accès Wifi et les antennes relais) (FenChong, 2012). Ces données sont téléchargées dans un fichier secret lors de la mise à jour du logiciel (du téléphone vers l'ordinateur). Une fois traitées par le ou les détenteurs, les données de géolocalisation sont de véritables mines d'information dont ils n'hésitent pas à se servir. Ainsi, les données sont recueillies sur «qui nous sommes, ce que nous savons, où nous avons été et où nous avons l'intention d'aller ». La quantité et le type des données collectées sont alors vastes : nos profils, nos dossiers, nos déplacements, nos recherches effectuées sur les sites Web, voire nos goûts et dégoûts, nos tweets, nos courriels, nos photos et vidéos (etc.) peuvent se retrouver sur la Toile, et cette liste ne cesse de s'accroître (WEF, 2011 ; FenChong, 2012 ; Olteanu-Raimond et al., 2012 ; Montjoye et al., 2013). Plusieurs études révèlent aussi l'impossible anonymisation véritable des données collectées dans leur forme actuelle. La collecte sur l'individu constitue une véritable source de profilage psychologique et comportemental (WEF, 2011). Au niveau des empreintes digitales, il faut douze points pour identifier sans équivoque une personne. Montjoye et al. (2013) indiquent de son côté que quatre données spatiales et temporelles sont aujourd'hui suffisantes pour nous distinguer dans une foule, ce qui dès lors peut nous laisser très craintif sur l'usage abusif qui est fait via les Smartphones.

La protection des personnes physiques à l'égard de l'utilisation et des traitements des données de géolocalisation demeure régie en France par les lois 2004-669 du 9 juillet 2004 et 2004-801/182 du 6 août portant sur les protections individuelles au sens large. Pour que des personnes non autorisées n’accèdent aux données, il est obligatoire de prendre des mesures de sécurité (accès à un site avec un identifiant et un mot de passe) selon la norme 51 de la CNIL. Cet usage est également protégé par un ensemble de lois associées au droit pénal (article 226-1 sur la protection de la vie privée; article 226-16 relatif aux droits des personnes résultant de traitements informatiques ; loi du 6 janvier 1978).

Face à ces abus, dont quelques exemples sont présentés ci-dessus, il existe en France une réglementation et des contraintes mais qui empêchent, en contrepartie, le 
développement d'applications. Les applications tentent de contourner les interdits depuis 2015 et la mise en place d'une loi portant sur le numérique (attendue pour 2016) est devenue grandement nécessaire. Par ailleurs, l'accord des personnes semble être le seul moyen à envisager si l'on souhaite utiliser librement des données d'itinérances issues des capteurs intégrés dans les Smartphones. Il ne faut pas non oublier que ce sont les individus eux-mêmes qui sont en cœur des dispositifs d'alerte, et qu'il faut donc les impliquer, les former, les tester... pour attendre d'eux les comportements attendus, ce que l'on a vite tendance à oublier.

\section{Comment créer une application utile, utilisable et utilisée alors ?}

\subsection{Une application à adapter à la demande citoyenne}

Toute nouvelle application répond à un besoin sociétal tout en tentant de pallier les lacunes des applications existantes. Au-delà du cadre juridique et réglementaire présenté auparavant, les avis des futurs usagers d'une application dédiée à l'alerte pour faire face au risque inondation ont été évalués lors de précédentes enquêtes menées dans le département du Vaucluse (très sensible aux crues rapides, même si les dramatiques crues de Vaison-la-Romaine de 1992 sont un peu lointaines dans le temps par rapport à des événements récents comme à Cannes le soir du 3 octobre 2015). Plusieurs recommandations ont ainsi été collectées, et c'est sur cette base que l'application AL'in (présentée juste après) a été conçue. Pour les répondants (354 questionnaires remplis), une application sur le risque inondation devrait avoir un caractère participatif (voire volontariste), intégrer les données de géolocalisation en temps réel, faire appel à l'appareil photographique pour valider ou non l'événement en cours (et ainsi éviter les mauvaises alertes) et devrait laisser de toute façon le choix à l'utilisateur pour l'installation (sur le téléphone ou vers une version en ligne via Q-R-Code). Une fois ouverte, on pourrait imaginer une interface simple et intuitive, qui proposerait à l'utilisateur un pré-formulaire (ce qui permet de le guider vers des choix préconstruits). D’autres technologies (la réalité augmentée ou la synthèse vocale) pourraient aussi être utiles. Après remplissage, le formulaire devrait être validé et le contenu transmis à un centre de décision (préconçu en amont ou localisé dans un service opérationnel), qui en retour pourrait diffuser l'alerte dans un rayon de quelques kilomètres à partir du point émetteur. La diffusion des alertes devrait par ailleurs se faire en dépit d'une coupure des antennes des opérateurs de téléphone mobile, et être adapté à tout type de Smartphone (même les plus anciens). Pour cela, il serait opportun de faire appel au mode " pair-à-pair », ce qui permettrait une connexion entre les Smartphones sans passer par les opérateurs via un système DTN (Delay Tolerent Network), procédé utilisé dans GeoCam par exemple. Les personnes entrant dans le périmètre de l'alerte (comme des touristes et des personnes qui sont en voiture par exemple) devraient eux aussi être informées des mesures à prendre et des gestes à adopter dans ce territoire qu'ils ne connaissent pas toujours sous l'angle des risques et notamment des inondations. 


\subsection{Un prototype transposable et interopérable}

Fort des recommandations émanant des futurs usagers potentiels, le prototype de l'application AL'in (Alerte aux Inondations) a été créé en suivant les principes d'une application Android (Gramlich, 2008 ; Conder et Darcey, 2011 ; Kumar, 2014). Ce prototype a été conçue (après une modélisation conceptuelle sous UML) à partir d'App Inventor ${ }^{1}$, une plateforme éducative d'apprentissage et de programmation propulsée par Google et le Massachusetts Institute of Technology (Bhagi, 2012 ; Shih et al., 2013). La plateforme fournit de nombreux composants de haut niveau facilitant l'utilisation des ressources du Smartphone, la communication avec les ressources web externes ou la manipulation de bases de données distantes ou stockées sur l'appareil : accéléromètre, appareil photo, envoi et réception SMS, GPS, enregistreur audio-vidéo, envoi et réception de messages Twitter, etc. (Wolber, 2010 ; Hsu et al., 2013).

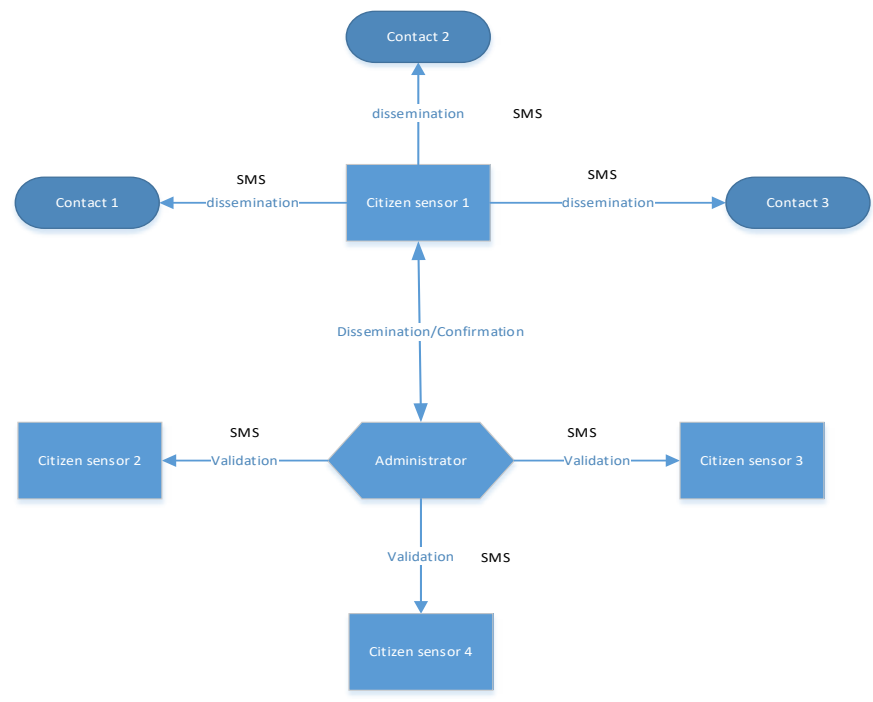

Figure 2. Principe général de fonctionnement du système

Fonctionnelle et opérationnelle, l'application s'appuie sur un procédé simple : l'utilisateur envoie des informations relatives à l'évènement qu'il observe (personnes en danger, intensité de la pluie, écoulements observés, route ou passages bloqués ou détruits) ou aux dommages (immeubles ou bâtiments endommagés) liés (figure 2), en remplissant un formulaire préconçu (pour guider l'usager et lui indiquer les informations à indiquer au plus vite) avec des questions et des réponses intuitives (indiquer la hauteur de l'eau à partir des membres du corps par exemple, sans se

1. http://ai2.appinventor.mit.edu/ 
baser sur des niveaux d'eau précis). En fonction de l'urgence de la situation et les conditions dans lesquelles le citoyen se trouve, cette information peut être détaillée ou synthétique (pour raccourcir le délai nécessaire à l'alerte). L’usager peut également associer une photo de l'événement, sans oublier les données de localisation qui lui sont fortement suggérées.

Après soumission du formulaire, les informations sont stockées dans une base de données externe (via Google fusion tables) permettant de les cartographier. Un SMS est diffusé à toutes les personnes que l'usager aura précédemment présélectionnées depuis sa liste de contacts (à l'image de ce que fait MyPublicAlert). Ce message contient un lien vers une cartographie présentant les évènements signalés (figures 3 et 4) et cette carte est accessible et consultable à tous même sans avoir reçu de SMS. Après l'avoir consulté, on peut consulter une liste de comportement à adopter. C'est l'alerte dite " passive » : il n'y a pas de réelle action d'intrusion dans le téléphone du citoyen, mais l'information est envoyée par une personne que l'on connait ou on fait la démarche d'aller la trouver (l'information) en consultant la carte (figure 4). C'est une façon de créer une certaine responsabilité citoyenne.

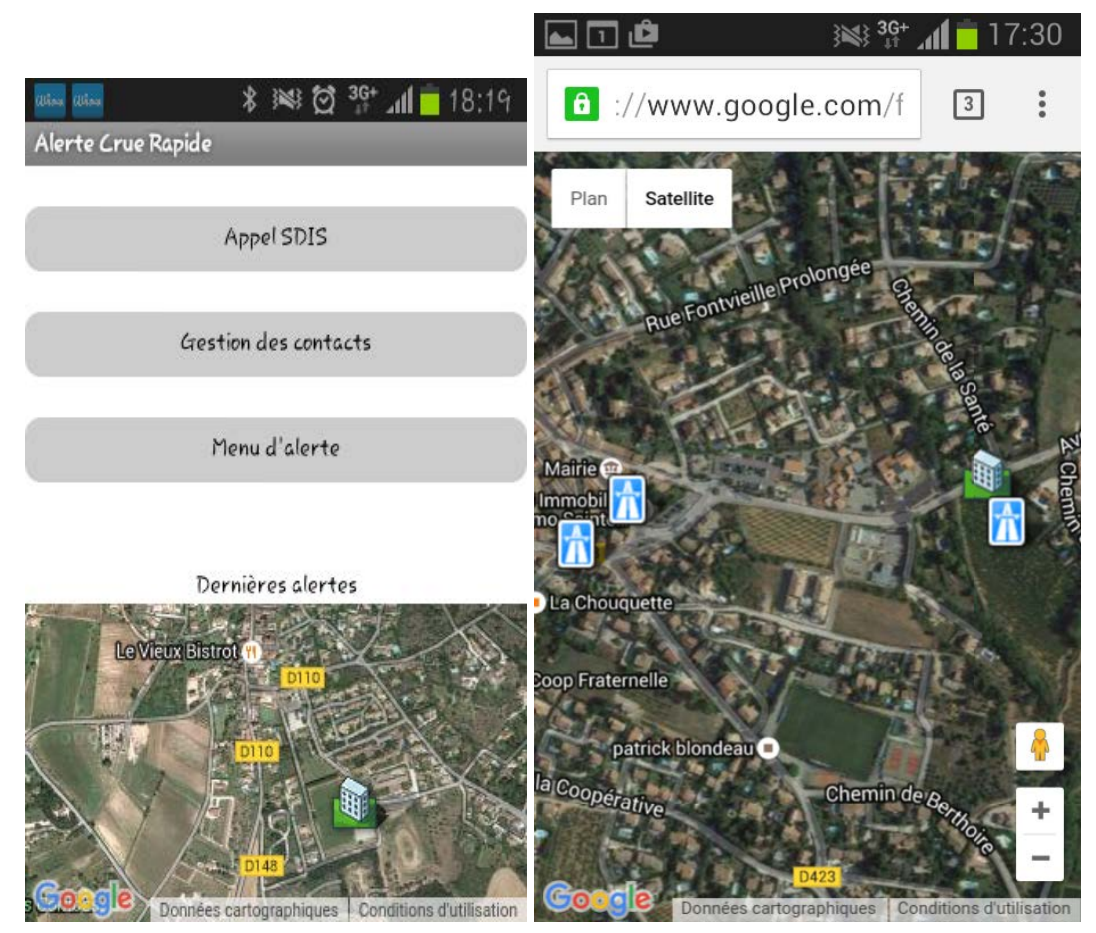

Figure 3. Écran principal, cartographie et icones utilisés dans l'application : personnes, immeuble, pluie, écoulement, 1 route 

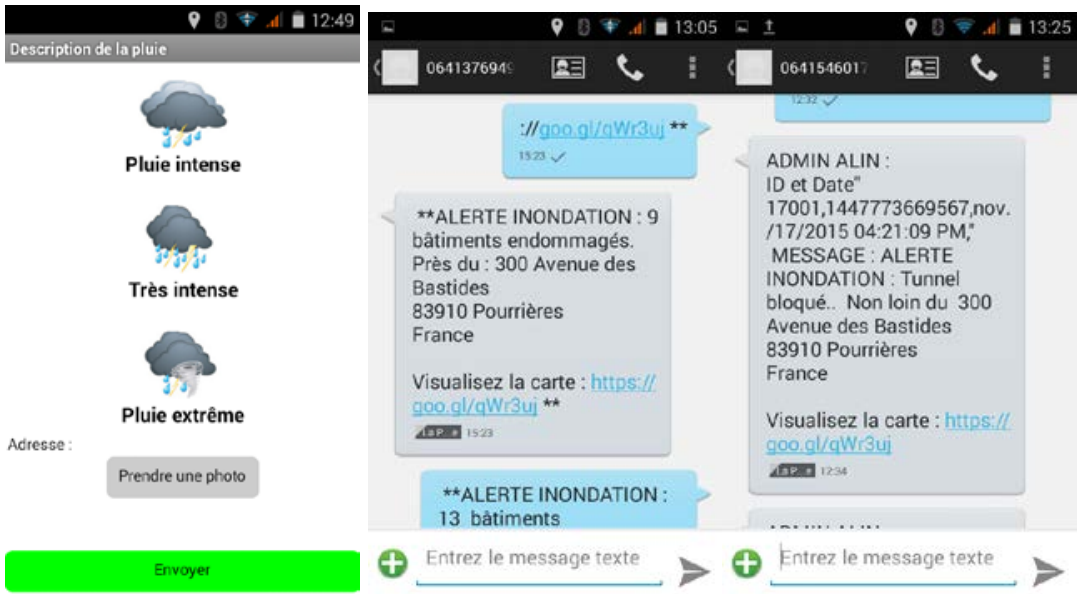

Figure 4. Écran pour qualifier la pluie observée (à gauche), voir la nature du SMS du citoyen (au centre) et le SMS reçu de l'administrateur (à droite)

Transmis également à l'administration, le SMS envoyé par le citoyen sera validé ou infirmé par un gestionnaire grâce à sa propre version de l'application pour une diffusion sur les mobiles des autres citoyens capteurs (ayant installé l'application) : c'est l'alerte « active ». L'évènement sera dès lors marqué de la mention « validé » et le SMS transmis aux autres citoyens portera l'entête " ADMIN ALIN » (figure 4). Cette version lui donne la possibilité de consulter dans l'ordre chronologique tous les messages transmis et de pouvoir déclencher ou non leur diffusion et de leur apposer ainsi le sceau administratif permettant aux citoyens qui vont le recevoir d'y accordé plus ou moins de l'attention et une certaine crédibilité (figure 5).

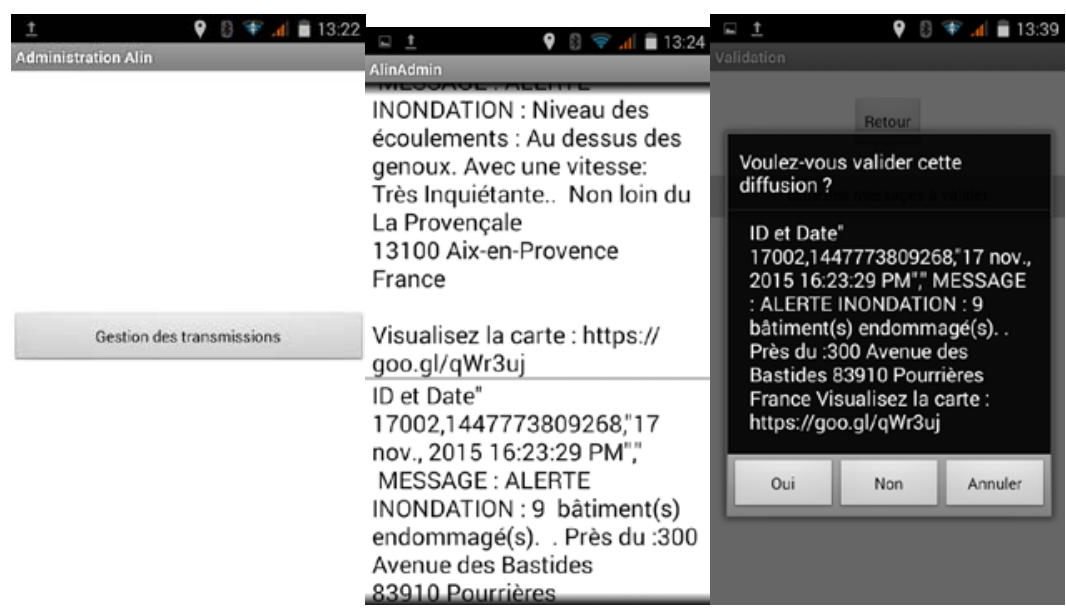

Figure 5. Version administrateur de l'application 


\subsection{Des expérimentations en cours et à poursuivre}

Pour vérifier la cohérence et le fonctionnement du prototype, l’application a été testée durant le mois de novembre 2014 dans deux communes : Cabrières d'Avignon (Vaucluse) et Pourrières (Var). Les retours de cette expérimentation (104 personnes enquêtées) sont intéressants car ils apportent des réponses concrètes tout en suggérant des points d'amélioration: la disponibilité du réseau mobile et la couverture internet sont parfois des handicaps dans ces territoires ruraux (pas toujours couvert par le réseau $2 \mathrm{G}$ à l'heure où les avancées technologiques sont au $5 G$, etc.) ; les autorités ont des difficultés à trouver une ou plusieurs personnes dédiées du côté administration car tout le monde n’a pas la «culture » Smartphone. Par ailleurs, le laps de temps dans lequel ce procédé atteint son objectif peut aller de quelques secondes à moins de 5 minutes pour une dizaine de personnes à contacter à partir d'un Smartphone si le réseau fonctionne correctement. Tout dépend en fait du nombre de personne à qui le Smartphone doit envoyer le SMS. Il est donc important de réfléchir à un algorithme permettant d'optimiser la transmission des messages.

Le système complet à envisager devra en outre présenter d'autres fonctionnalités clés : une application à la fois hybride et multiplateforme (HTML5, Android et iOS). Pouvoir s'affranchir aujourd'hui des contraintes liées aux systèmes d'exploitation permet d'assurer une pérennité et un usage sur du long terme de l'application. On pourra donc renseigner et alimenter le système sans avoir besoin d'installer une application spécifique sur son smartphone. Envisager un serveur cartographique, une base de données ainsi qu'un serveur SMS autonomes sont aussi des éléments dont il faut tenir compte.

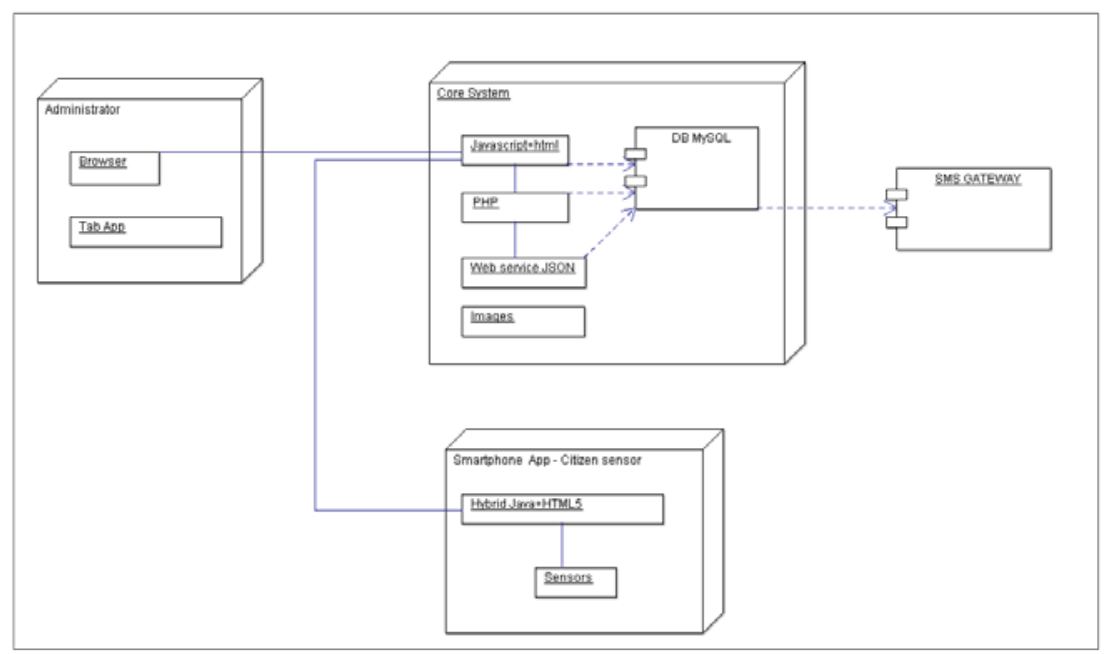

Figure 6. Architecture globale du futur système (diagramme de déploiement) 
Intégrer ces fonctionnalités dans l'application nécessite préalablement de passer par une importante phase conceptuelle qui permettra de modéliser du mieux possible la solution à laquelle on souhaite aboutir (Morand, 2007). UML (Unified Modeling Language) est un langage de modélisation des systèmes dont l'un des diagrammes (Uses Case) permet de modéliser les interactions entre le système et les acteurs impliqués. Comme nous souhaitons que les citoyens contribuent volontairement à l'alerte et dans un délai très raisonnable, il était important de pouvoir modéliser le comportement de ces derniers et des individus susceptibles de recevoir les alertes diffusées. Par ailleurs, plusieurs travaux ont déjà démontré l'efficacité et la valeur ajoutée non négligeable qu'apporte l'usage de l'approche UML pour la réalisation d'applications sur Smartphones (Haugsrud, 2009 ; Kraemer, 2011 ; Parada et al., 2011, 2012 ; Quanyin et al., 2012 ; Stuurman, 2014). La mise en œuvre d'UML dans le cadre de ce travail a abouti à un diagramme de déploiement cohérent (figure 6).

La réalisation du diagramme de déploiement a nécessité en amont la création du diagramme d'utilisation, du diagramme d'activités, du diagramme d'états ainsi que du diagramme de classes et de séquences. Présentons ici le diagramme d'états (figure 7). Basé sur trois notions (état, événement et comportement), ce diagramme a pour but d'offrir une vision non ambiguë de l'ensemble des comportements de l'élément auquel il est attaché. Un état est une situation que l'on peut qualifier de stable dans la vie d'un objet et dans laquelle il effectue une activité ou il attend un événement. L'évènement pour sa part est en fait une transition déterminant le passage de l'objet d'un état à un autre. Quant au comportement, il se présente le plus souvent sous deux formes : les actions pouvant être interrompues (i.e les activités) et celles ne pouvant l'être (i.e les traitements).

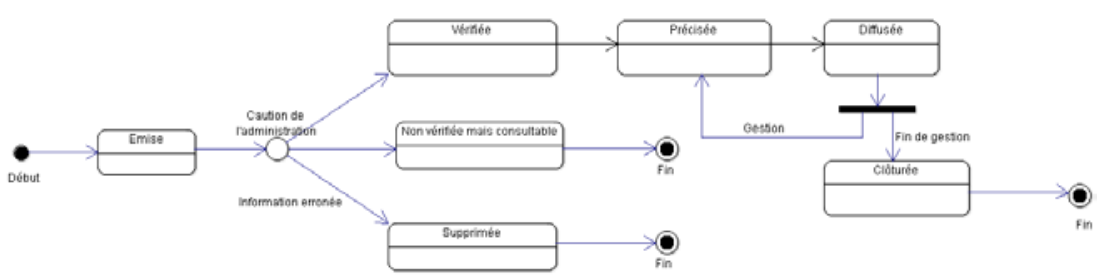

Figure 7. Diagramme d'état relatif à une alerte citoyenne émise

Réaliser un diagramme d'état suppose l'identification d'un objet, une entité ou une unité du système dont la nature et donc l'état évoluent au cours des interactions entre les différents acteurs. Dans le cas d'Al'in, la principale entité dont la nature évolue au cours du fonctionnement du système est l'information émise par le citoyen capteur. En effet, celle-ci passe par plusieurs phases depuis sa création jusqu'à sa diffusion par l'administrateur. L'utilisateur qui installe l'application puis s'en sert peut lui aussi être considéré comme une entité, « une entité » dont la nature peut évoluer tout au long du processus relatif à la diffusion de cette information. 


\section{Conclusion}

Face aux caractéristiques spatiales $\left(<10 \mathrm{~km}^{2}\right)$ et temporelles (de $1 \mathrm{~h}$ à $6 \mathrm{~h}$ ) des crues rapides, plus vite la collecte et le traitement des données sera effectuée, plus efficace sera l'alerte et in fine les opérations de secours. L'application proposée ici se veut participative, volontariste et gratuite pour tout le monde. Elle pourrait être utilisée pour contribuer à réduire les écarts entre les services de l’Etat (qui gardent la mainmise sur l'alerte) et les citoyens qui se sentent dépourvus d'outils en cas de crues aussi virulentes qu'imprévisibles. Les problèmes liés à la responsabilité et à la législation concernant l'information ne se posent pas vraiment lorsqu'il s'agit d'informations sous le sceau de l'anonymat. Les choses deviennent en revanche plus compliquées lorsqu'il s’agit d'une personne envoyant une information bien précise demandant par exemple une assistance. Ainsi, même une seule information d'alerte provenant d'un seul endroit peut s'avérer utile à un moment donné.

Au regard de la rapidité des développements technologiques, du potentiel et des services offerts aux utilisateurs, les résultats encourageants ne doivent toutefois pas faire oublier les réserves qui subsistent. A ce titre, nous pouvons reprendre la citation de B. Stiegler (2008) qui, parlant de ces techniques, évoquait leur aspect ambivalent, à la fois «remède et poison ». Tout le monde n'utilise pas son Smartphone de façon assidue et alerter une personne dont le téléphone n’est pas allumé est problématique. Par ailleurs, bien que les opérateurs privés aient pour objectifs une couverture 4G de 99,6 \% de la population française à l'horizon 2027, la couverture actuelle se situe aux alentours de 25 \% (ARCEP, 2015). Le maillage est donc encore très irrégulier, ce qui pose problème, d'autant plus dans les petits bassins situés en montagne et qui sont justement sujets aux crues rapides. S’ajoute à ce constat somme toute assez négatif les problèmes d'endommagement des matériels (antennes, réseau électrique, etc.), inévitables lors d'évènements hydrométéorologiques, qui constituent des points de vulnérabilité des systèmes RSN et Smartphones.

Compte tenu du manque de dispositions légales précises autour de ces questions, il est préférable de considérer l'outil proposé comme élément pouvant contribuer à améliorer les dispositifs actuels. L'introduction des technologies numériques dans le domaine de l'alerte est récente et demande donc une appropriation de ces systèmes par les politiques en place et par la population (pourquoi pas, dans une démarche coconstruite entre tous). C'est la raison pour laquelle l'amélioration de l'alerte face aux crues rapides viendra plus d'une approche systémique que d'une solution technique (d'autant plus si elle est imposée pour tous sans aucun accompagnement).

\section{Remerciements}

Les auteurs tiennent à remercier pour leur soutien financier la Région PACA, le bureau d'études Risques \& Développement (Eric Leroi), la Fédération de recherche Agor@ntic (FR 3612 CNRS) et l'université d'Avignon et des Pays de 
64 ISI. Volume $21-n^{\circ} 4 / 2016$

Vaucluse. Les auteurs remercient également les relecteurs pour leurs remarques constructives, et Florence Sèdes pour l'organisation de ce numéro thématique.

\section{Bibliographie}

Artigue G., Johannet A., Borrell V. et S. Pistre (2012). Flash flood forecasting in poorly gauged basins using neural networks: case study of the Gardon de Mialet basin (southern France). Nat Hazards Earth Syst Sci, n 12, p. 3307-3324.

Autorité de Régulation des Communications Électroniques et des Postes (2015). Les obligations de déploiement 4G (LTE). Récupéré du site de l’ARCEP le 2 juillet 2015 : http://www.arcep.fr/sides/index.php?id=8161

Bhagi A. (2012). Android Game Development with AppInventor. Memoire de Master of Engineering in Electrical Engineering and Computer Science, MIT, Consulté le 04/02/2016: http://appinventor.mit.edu/explore/sites/all/files/Resources/Thesis_ FINAL_ AnshulBhagi.pdf.

Conder S., Darcey L. (2011). Android wireless application development, Developer's library.

Créton-Cazanave L. (2010). Penser l'alerte par les distances: entre planification et émancipation, l'exemple du processus d'alerte aux crues rapides sur le bassin versant du Vidourle, thèse de Géographie, Université de Grenoble.

Delahaye D. (2002). Apport de l'analyse spatiale en géomorphologie. Modélisation et approche multiscalaire des risques. Mémoire d'habilitation à diriger des recherches. Université de Rouen.

Douvinet J., Denolle A.S., Defossez S., Anselle A (2011). Les maires face aux Plans de Prévention du Risque Inondation (PPRI). L'espace géographique, n 1, p. 31-46.

Douvinet J., Saint-Martin C., Le Bihan G., Kouadio J., Payrastre O., Vinet F., Pons F., Moncoulon D., Janet B., Mermet E., Fouchier C., Javelle P. (2016). A flash flood warning system over small-ungauged basins integrating damage dimension in France: supports and challenges, Proceedings of FloodRiks, Lyon, septembre.

Fen-Chong J. (2012). Organisation spatio-temporelle des mobilités révélées par la Téléphonie mobile en Ile-de-France. Thèse en Géographie, Université Paris 1.

Garambois P. A., Roux H., Larnier K., Dartus D. (2011). Relations between streamflow indices, rainfall characteristics and catchment physical descriptors for flash flood events. Proceedings of a symposium held Weather Radar and Hydrology, 2011, Exeter.

Gaume E., Bain V., Bernardara P., Newinger O., Barbuc M., Bateman M., Blaškovičová M. et al. (2009). A Compilation of Data on European Flash Flood, Journal of Hydrology, $\mathrm{n}^{\circ} 367$, p. $70-79$.

Goodchild M.F (2007). Citizens as sensors: the world of volunteered geography. GeoJournal, vol. $69, n^{\circ} 4$, p. $211-221$.

Gramlich N. (2008). Android Programming, Electronic Book.

Gupta A., Lamba H., Kumaraguru P., Joshi A. (2013). Faking Sandy: characterizing and identifying fake images on Twitter during Hurricane Sandy. $22^{\text {nd }}$ International Conference on World Wide Web Conference, Rio de Janeiro. http://dl.acm.org/citation.cfm?id =2488033 
Haugsrud S. (2009). Developing Android Applications with Arctis. Memoire de Master en Science in Communication Technology. Norwegian University of Science and Technology.

Hebert M. (2014). Les technologies smartphones peuvent-elles aider en cas d'alerte aux crues rapides ? Réflexion à l'échelle des départements du Vaucluse et du Var. Mémoire de Master 1 de géographie, Université d'Avignon et des Pays de Vaucluse.

Hsu Y.-C., Ching Y.-H. (2013). Mobile app design for teaching and learning: Educators' experiences in an online graduate course. The International Review of Research in Open and Distance Learning, vol. 14, ${ }^{\circ}$ 4, p. 117-139.

Jonkman S. (2005). Global perspectives on loss of human life caused by floods. Natural Hazards, vol. 34, n², p. 151-175.

Jonkman S.N., Kelman I. (2005). An analysis of causes and circumstances of flood disaster deaths. Disasters, vol. 29, n 1, p. 75-97.

Kouadio J., Douvinet J., Grasland (2014). Utiliser les Smartphones comme outils d'aide face aux crues rapides: enjeux, contraintes et limites. Actes du colloque SAGEO, Colloque International de Géomatique et d'Analyse spatiale, 24-27 novembre, p. 287-292.

Kraemer F. A. et al. (2011). Engineering android applications based on UML activities. Proceedings of the $14^{\text {th }}$ international conference on Model driven engineering languages and systems.

Kuma R. (2014). Application Development in Android. International Journal of Advanced Research in Computer and Communication Engineering, vol. 3, $\mathrm{n}^{\circ} 6$.

Laurila J. K., Gatica-Perez D., Aad I., Blom J., Bornet O., Minh Tri Do,M., Dousse, O., Eberle J. et Miettinen M. (2013). From big smartphone data to worldwide research : the mobile data challenge. Journal Persuasive and Mobile Computing, vol. 9, $\mathrm{n}^{\circ} 6$, p. 752-771. doi : 10.1016/j.pmcj.2013.07.014

Lemmens J. S., Valkenburg P.M., Peter J. (2009). Development and validation of a game addiction scale for adolescents. Media Psychology, vol. 1, n 12, p. 77-95.

Mathys N., Peteuil C. (2012). Synthèse et analyse des données de crues des bassins versants des Alpes françaises, Conference Proceedings $12^{\text {th }}$ Congress INTERPRAEVENT 2012, Grenoble.

Mobile Marketing Association (2015). Le Baromètre Trimestriel - du Marketing Mobile en France, Rapport de recherche MMA, mars.

Montjoye Y.-A., Hidalgo C.A., Verleysen M., Blondel V.D. (2013). Unique in the Crowd:The privacy bounds of human mobility, http://www.nature.com/articles/srep01376

Moran B. (2007). Le logiciel, sujet et objet de la norme. Droit et Société, n 65, p. 41-51.

Morss R.E. et al. (2016). How do people perceive, understand, and anticipate responding to flash flood risks and warnings? Results from a public survey in Boulder, Colorado, USA. Journal of hydrology. http://dx.doi.org/10.1016/j.jhydrol.2015.11.047

Object Management Group (2015). Unified Modeling Language, Version 2.5, OMG, http://www.omg.org/spec/UML/2.5/PDF

Olteanu-Raimond A.-M., Couronné T.,cFen-Chong J., Smoreda Z. (2012). Modélisation des trajectoires spatio-temporelles issues des traces numériques de téléphones portables. Le Paris des visiteurs, qu'en disent les téléphones mobiles ?, Revue Internationale de Géomatique, vol. 22, n 3, p. 413-437, doi:10.3166/rig.22.413-437 
Parada A.G., Siegert E., de Brisolara L.B. (2011). Generating Java Code from UML Class and Sequence Diagrams. Brazilian Symposium Computing System Engineering (SBESC), doi:10.1109/SBESC.2011.22.

Parada A. G. et de Brisolara L. B. (2012). A model driven approach for Android applications development. Brazilian Symposium on Computing System Engineering (SBESC).

Quan-yin Z., Yin J., Chengjie X., Rui G. (2011). A UML Model for Mobile Game on the Android OS. International Conference on Advances in Engineering.

Ruin I., Gaillard J.-C., Lutoff C (2007). How to get there? Assessing motorists’ flash flood risk perception on daily itineraries. Environ. Hazards, vol. ${ }^{\circ}$, n ${ }^{\circ} 3$, p. 235-244.

Ruin I., Creutin J. D., Anquetin S., Gruntfest E., Lutoff C. (2008). Human vulnerability to flash floods: Addressing physical exposure and behavioral questions. In Flood risk proceedings of the European Conference on Flood Risk Management Research into Practice management, Oxford.

Saint-Martin C. (2014). Intégration, au système d'avertissement de la méthode AIGA, du facteur d'exposition des territoires au risque inondation. Rapport de stage effectué au sein de IRSTEA en vue de l'obtention du grade de Master Spécialité : Gestion des Catastrophes et des Risques Naturels (G.C.R.N.).

Shih F., Seneviratne O., Miao D., Licardi I., Kagal L., Patton E.W., Castillo C., Meier P. (2013). Democratizing mobile app development for disaster management. In: Proceedings of the $2^{\text {nd }}$ Workshop on Semantic Cities.

Slingsby A., Beecham R. et Wood J. (2013). Visual analysis of social networks in space and time using smartphone logs. Journal Persuasive and Mobile Computing, vol. ${ }^{\circ} 9, n^{\circ} 6$, p. 848-864. doi: 10.1016/j.pmcj.2013.07.002

Smith A., Rainie L., McGeeney K., Keeter S., Dugan M.(2015). U.S. Smartphones use in 2015 report. Récupéré du site du Pew Research Center le 25 juin 2015 : http://www.pewinternet.org/files/2015/03/PI_Smartphones_0401151.pdf

Stiegler B. (2008). Réenchanter le monde. La valeur esprit contre le populisme industriel, Flammarion, Paris.

Stuurman S., van Gastel B. E., Passier H. J. M. (2014). The design of mobile apps: what and how to teach? Proceedings of the Computer Science Education Research Conference.

Vinet F. (2010). Le risque inondation. Diagnostic et gestion, Lavoisier.

Wolber D. (2010). A blocks language for mobile phones: App Inventor for Android. In E. Canessa \& M. Zennaro (Eds.), mScience: Sensing, computing and dissemination. Trieste, Italy: ICTP- The Abdus Salam International Centre for Theoretical Physics.

World Economic Forum (2011). Personal Data: The Emergence of a New Asset Class, WEF.

Woyke E. (2014). The Smartphone: Anatomy of an industry, The new press, New York. 